\title{
Performance analysis and optimization for non-uniformly deployed mmWave cellular network
}

\author{
Xuefei Zhang ${ }^{1 *} \mathbb{D}$, Yinjun Liu' ${ }^{1}$, Yue Wang ${ }^{1}$ and Juan Bai ${ }^{2}$
}

\begin{abstract}
In this paper, we propose a multi-tier mmWave cellular framework where sub-6 GHz macro BSs (MBSs) are assumed as a Poisson point process (PPP) and small-cell BSs (SBSs), operating on either mmWave or sub- $6 \mathrm{GHz}$, follows non-uniform Poisson cluster point (PCP) model. This paper proposes both centralized and distributed user association algorithms. For the centralized two-step algorithm, we aim to maximize the sum rate while satisfying quality of service (QoS) and power consumption constraints based on eigenvalue analysis. Then, we derive the association probability, the coverage probability, and the average achievable rate, cosidering directivity and blockage effect, by stochastic geometry. On this basis, a distributed user association algorithm is proposed. The simulation results demonstrate the accuracy of our theoretical analysis and also reveal the effect of some parameters on the network performance. In addition, the proposed centralized algorithm can achieve near-optimal sum rate with a low complexity.
\end{abstract}

Keywords: MmWave cellular network, Non-uniform distribution, Stochastic geometry

\section{Introduction}

Nowadays, the fifth generation mobile communication network (5G) are undoubtedly one of the most attractive topics in both academic and industrial fields. Many res earchers engage themselves to explore the new advanced methodologies and technologies in 5G, to support the booming data traffic with reduced energy consumption and improved quality of service (QoS) provision. Some enabling technologies, such as heterogeneous networks (HetNets), massive multiple-input multiple-output (MIMO), and millimeter wave (mmWave) techniques, have been identified to bring $5 \mathrm{G}$ to fruition [1].

MmWave communications, which operate from $30 \mathrm{GHz}$ to $300 \mathrm{GHz}$, have attracted much attention due to the shortage of microwave frequency [2]. It can provide the larger bandwidth, which can support the higher data rate transmission. Besides, mmWave communications have no effect on the traditional cellular communication in sub$6 \mathrm{GHz}$, since they operate at the higher and different frequencies. Therefore, the deployment of mmWave BSs

\footnotetext{
*Correspondence: zhangxuefei@bupt.edu.cn

${ }^{1}$ National Engineering Laboratory for Mobile Network Technologies, Beijing

University of Posts and Telecommunications, 100876 Beijing, China

Full list of author information is available at the end of the article
}

not only offloads the data traffic of existing cellular frequency, but also reduces the interference. However, a major channel characteristic of mmWave communications is its sensitivity to the blockages, which is a serious problem due to the poor performance of mmWave links at penetrating into or diffracting around solid objects [3]. Therefore, mmWave communication is commonly used in short-range directional transmission with directional antennas instead of isotropic antennas. On the other hand, the rigorous directivity and high penetration losses lead to the fact that mmWave systems are noise-limited. Therefore, the user association algorithms designed for the interference-limited networks cannot be directly applied in the mmWave systems [4].

Due to the two mentioned fundamental differences from conventional sub-6 GHz cellular systems, the channel models of mmWave communications should be modeled carefully. Some research proposed the mmWavepattern models including the effects of blocking, directional beamforming, small-scale fading, and path loss for line-of-sight (LOS) and non-line-of-sight (NLOS) links [3-5]. Specifically, the blockages, resulting in the serious power loss at the high mmWave frequencies, are assumed stationary and isotropic [5, 6]. For mathematical
Springer Open

(c) The Author(s). 2019 Open Access This article is distributed under the terms of the Creative Commons Attribution 4.0 International License (http://creativecommons.org/licenses/by/4.0/), which permits unrestricted use, distribution, and reproduction in any medium, provided you give appropriate credit to the original author(s) and the source, provide a link to the Creative Commons license, and indicate if changes were made. 
tractability, a LOS ball model was proposed for simplifying blockage modeling where BSs inside the LOS ball were considered to be in LOS whereas any BS outside of the LOS ball was treated as NLOS [6]. In [7], this blocking model was modified by adding a LOS probability within the LOS ball, and this approach was shown to reflect several realistic blockage scenarios. Meanwhile, the pathloss exponent is considered higher in NLOS links (e.g., commonly about 4) than that in LOS links (e.g., around 2) [8]. Furthermore, some real data is obtained to verify the parameters of some blocking model (e.g., real building data in the UT Austin, downtown LA regions, NYC, and Chicago $[4,6])$. It is revealed that the environment has the effect on the block behaviors, and the blocking models should be carefully selected according to the propagation environment (e.g., 3GPP-like models could be sufficient to fit the urban regions; generalized LOS ball model gives a good fit for dense random deployment of BSs, etc. [4]).

Meanwhile, the directional beamforming, for compensating the increased path loss at mmWave frequencies and for overcoming the more serious noise due to the large transmission bandwidth, is approximated by considering a sectored antenna model $[9,10]$. In [9], mmWave BSs are equipped with directional antennas, and UEs perform the perfect beam alignment, whereas in [10], both the users and mmWave BSs are assumed to estimate the angles of arrival and to adjust their antenna orientations accordingly. Moreover, large antenna arrays, enabling massive MIMO and hybrid beamforming techniques, can contribute to the directive transmission. The rate of multiuser MIMO with hybrid beamforming [11] or with user pairing [12] or joint spatial division and multiplexing [13] in mmWave network are analyzed.

\section{Methods/experimental}

Motivated by the mathematical tractability, stochastic geometry has been accepted as a popular and useful tool to analyze performance of HetNets, and some significant results and conclusions are obtained [14-19]. Stochastic geometry is introduced into the mmWave network analysis where locations of both sub-6 GHz and mmWave BSs are considered as Poisson point process (PPP), and block effects are incorporated into the system model [20]. The expressions, or upper and lower bound of some performance metrics (e.g., coverage probability, outage probability, average rate, etc.) are derived mostly under PPP-based mmWave network scenario $[3,4,6,9,10]$. Actually, as one of capacity-increasing techniques, mmWave BSs commonly work along with the cellular network and are distributed only on some hot spot areas. Therefore, non-uniform location distribution of mmWave BS is more appropriate to model the realistic scenario. To the best of our knowledge, few works adopt the non-uniform mmWave BS location distribution model, which will be the main difference of our paper from previous papers. In this paper, we make centralized and a distributed user association analysis for mmWave cellular system. The contributions of this paper can be summarized as

- We propose a centralized two-stage user association and power control algorithm to maximize the network sum rate while satisfying SINR and power consumption constraints. The optimization problem is divided into user association and power control subproblems. Specifically, for user association, the eigenvalue analysis is performed to drop communication links successively that cause the maximum sum of the interference power in the network until the feasibility condition is satisfied. In addition, binary power (BP) allocation is applied to solve the optimal power allocation.

- We derive analytical expressions on the association probability, the coverage probabilities, and the average achievable rate, considering directivity and blockage effect, using stochastic geometry. On this basis, a distributed distance-based user association method is proposed to decide the pattern of BSs (conducting in either sub-6 GHz or mmWave).

The remainder of this paper is organized as follows. In Section 3, the system model for mmWave cellular networks is described. The proposed centralized algorithms are presented in Section 4. In Section 5, the analytical expressions for the association probability, coverage probability, and the average achievable rate are derived. Further, a distributed distance-based user association method is proposed to decide the patterns of BSs. Simulation results are provided in Section 6 to compare the performance of the proposed algorithms, which are followed by our conclusions in Section 7.

\section{System model}

We consider a downlink mmWave cellular network. Specifically, macro base stations (MBSs) are uniformly distributed in $\mathbb{R}^{2}$ according to Poisson point process (PPP) with density $\lambda_{m u}$; small-cell base stations (SBSs) are generated by Poisson cluster process (PCP) $\Phi_{s}$ where the parent points following PPP with density $\lambda_{p}$ forms the centers of these clusters and the daughter points (i.e., SBSs) are uniformly distributed around the cluster center within the radius $R$ [19]. The number of daughter points within each cluster is $c$. In a hybrid deployment, MBSs, under sub$6 \mathrm{GHz}$, are essential to provide wide coverage to guarantee a consistent service for UEs whereas mmWave may be used in SBSs mainly to deliver high rate to individual UEs. Specifically, all MBSs and some SBSs share sub-6 GHz, whereas the rest of SBSs operate at the mmWave bands. It is worth noting that SBSs in one cluster will choose the 
same pattern, i.e., either sub- $6 \mathrm{GHz}$ or mmWave. Without loss of generality, we assume that a SBS cluster is under sub-6 GHz with probability $p_{s u}$, i.e, the density of sub$6 \mathrm{GHz}$ SBSs $\lambda_{\mathrm{su}}=c p_{\mathrm{su}} \lambda_{p}$; otherwise, it conducts under mmWave, i.e, the density of mmWave SBSs $\lambda_{\mathrm{sm}}=c p_{\mathrm{sm}} \lambda_{p}$ where $p_{\mathrm{sm}}=1-p_{\mathrm{su}}$. Thus, we regard BS location distribution as three independent tiers, i.e., sub-6 GHz MBS tier, sub-6 GHz SBS tier, and mmWave SBS tier, as is shown in Fig. 1.

The two main characteristics of mmWave transmission are blockage effect and directivity at transmitter and/or receiver.

(1) The actual mmWave transmission directivity is approximated by a sectored model $[3,4,6,9,10]$, where a UE receives a signal with directivity gain $G_{\max }$ if the UE's angle $\theta$ with respect to the best beam alignment is within the main beamwidth $2 \omega$ of the serving mmWave cell and with directivity gain $G_{\min }$ otherwise. This is formulated by

$$
G(\theta)=\left\{\begin{array}{l}
G_{\max }|\theta| \leq \omega \\
G_{\min } \text { otherwise }
\end{array}\right.
$$

whereas $G(\theta)=1$ under sub- $6 \mathrm{GHz}$ cell. Due to the fact that $\theta$ is independent of all other variables, we simplify the $G(\theta)$ as $G_{j}$ for the $j$-tier directivity gain.

(2) We approximate the blockage effect by a modified LOS ball model under mmWave link [4], i.e., a UE within a distance $D$ to an mmWave SBS is LOS link, otherwise is considered NLOS link.
Thus, the received SINR at the typical user (at origin $o$ ) is

$$
\operatorname{SINR}_{k}(x)=\frac{P_{k} G_{k} h_{x}\|x\|^{-\alpha_{k}}}{I_{\Phi \backslash x}(x)+N_{0}}
$$

where $x$ is the location of the associated BS, $\Phi$ is the set of all BSs' locations, $h_{x}$ is the small-scale fading coefficient between UE at origin and BS at $x$ and is assumed to be independent and identically distributed (i.i.d.) Rayleigh fading [9]. The path loss exponent of an mmWave link depends on the link distance whereas the path loss exponent of a sub-6 GHz link is independent of the link distance. Specifically, the path loss exponent $a_{k}$ equals $a_{s}$ if the link is under sub-6 GHz, equals $a_{l}$ if the link is LOS under mmWave and equals $a_{n}$ otherwise. The additive noise is complex Gaussian distributed [19]. The transmit power of MBSs, SBSs operating at sub- $6 \mathrm{GHz}$, and SBSs operating at mmWave frequency are $P_{\mathrm{mu}}, P_{\mathrm{su}}, P_{\mathrm{sm}}$, respectively. The aggregated interference for UE at $x$ can be expressed as $[4,21]$

$I_{k, \Phi \backslash x}(x)=\left\{\begin{array}{cc}\sum_{j \in\{\mathrm{mu}, \mathrm{su}\}} \sum_{z \in \Phi_{j} \backslash x} P_{j} G_{j} h_{z}\|z\|^{-\alpha_{j}} & k \in\{\mathrm{mu}, \mathrm{su}\} \\ 0 & k \in\{\mathrm{sm}\}\end{array}\right.$

where $\Phi_{j}$ represents the set of $j$-tier BS locations and

- $k=$ mu means that $k$ tier is sub-6 GHz macro BS tier.

- $k=\mathrm{s}$ means that $k$ tier is sub-6 GHz SBS tier.

- $k=$ sm means that $k$ tier is mmWave SBS tier.

The conditions that UE associates with MBS tier, sub$6 \mathrm{GHz}$ SBS tier and mmWave SBS tier are illustrated in

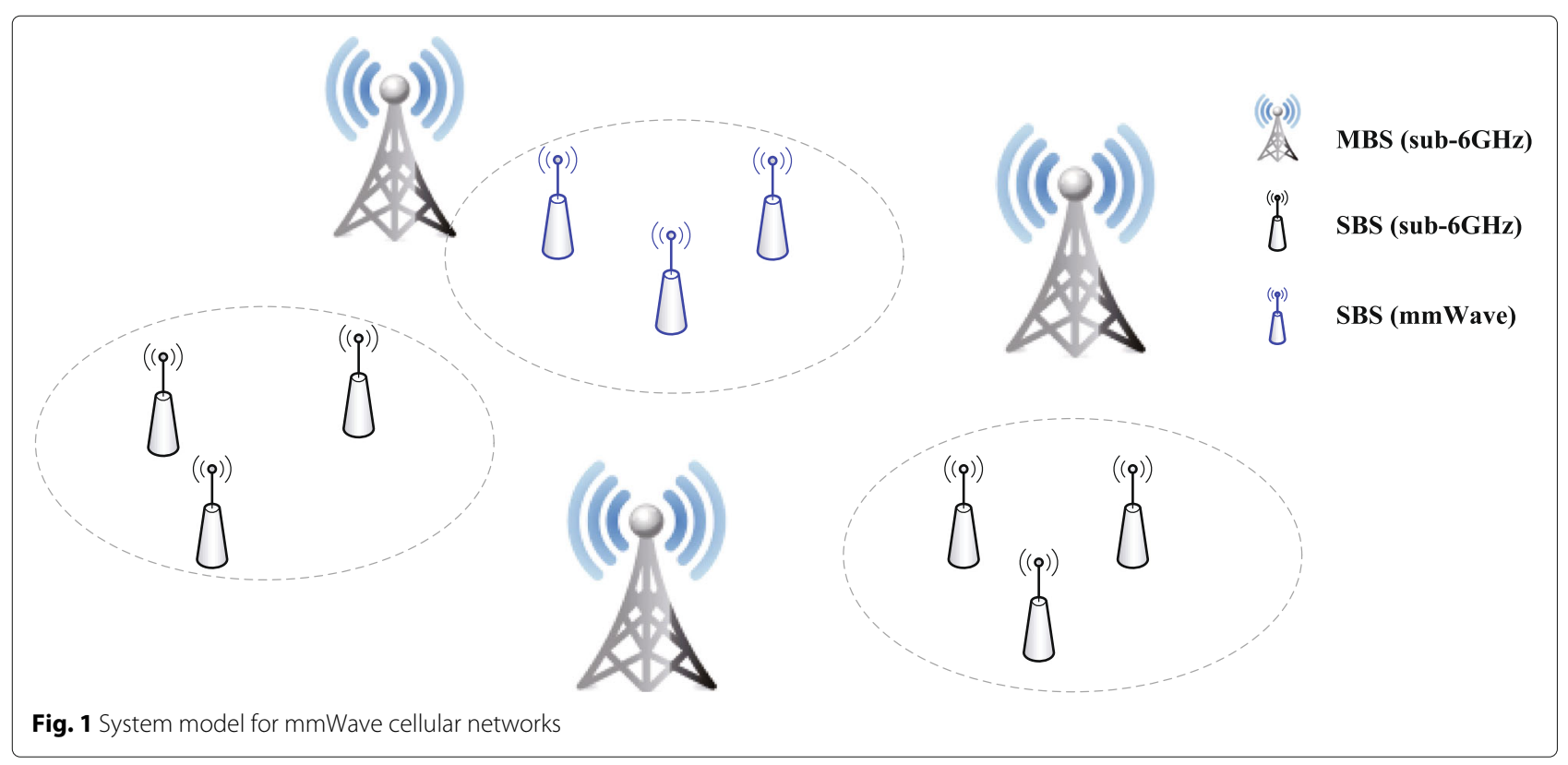


Figs. 2, 3, and 4, respectively. Unlike sub-6 GHz networks, mmWave cellular systems will be noise-limited due to the directivity and blocking effects discussed earlier, in conjunction with the large bandwidth which brings in much more noise power $[4,9]$. In such cases, the SNR distribution can be used as an approximation of the SINR. This is in contrast to sub- $6 \mathrm{GHz}$ cellular networks, which are often interference-limited, meaning SIR $\approx$ SINR instead.

In this paper, we consider an mmWave cellular network in which users intend to communicate with the BS in either mmWave or sub-6 GHz band. In this mmWave cellular system, we propose a centralized and a distributed user association algorithm, respectively. In Section 4, we propose a centralized user association and power control algorithm. The main idea of the centralized algorithm is to design the user association and transmit power of users to maximize the network sum rate while satisfying the individual target SINR constraints for all links. Note that the centralized algorithm depends on the global channel state information (CSI) possibly at a centralized controller, which is significant to the power design. Note that the centralized algorithm requires global channel state information (CSI) possibly at a centralized controller, which may incur high CSI feedback overhead. To resolve this issue, we propose a distributed user association method in Section 5, which requires distance about the link between the transmitter and its corresponding receiver only. The main goal of the distributed algorithm is to maximize the coverage probability, thereby the basis of which is to calculate the coverage probability. Therefore, we derive the coverage probability as well as average achievable rate in Section 5. Moreover, the probability distribution function (pdf) of associated distance and the association probability are also derived in Section 5 , which are the necessary components of the expression of coverage probability. Thus, we analyze the mmWave cellular network performance from the perspectives of centralized and distributed control.

\section{A centralized two-stage user association and power control algorithm}

A main feature of mmWave cellular networks is that the mmWave links are managed by MBSs in a centralized manner. Commonly, the centralized algorithm can provide the upper performance bound for its distributed counterpart. In this section, we suppose that MBS is able to acquire global channel state information (CSI). Based on the system model in Section 2, we generate a mmWave cellular network of $N$ sub-6 GHz MBSs and $M$ mmWave SBSs. On this basis, we would like to maximize the network sum rate under SINR and power consumption constraints. The optimization problem can be formulated as

$$
\begin{aligned}
& \max _{\mathbf{P}} \sum_{i=1}^{M+N} \log \left(1+\operatorname{SINR}_{i}(x)\right), \\
& \text { s.t. }(\mathbf{I}-\mathbf{F}) \mathbf{P} \geq \mathbf{b}, \\
& 0 \leq P_{i} \leq P_{\mathrm{mu}}^{\max }, i=1,2, \cdots, N, \\
& 0 \leq P_{i} \leq P_{\mathrm{sm}}^{\max }, i=N+1, N+2, \cdots, N+M .
\end{aligned}
$$

where (4) corresponds to the SINR requirement to be satisfied at all $M+N$ receivers. All $M+N$ inequalities are put together in a matrix-form inequality, as is shown in (4). The element in the normalized gain matrix $\mathbf{F}$ is

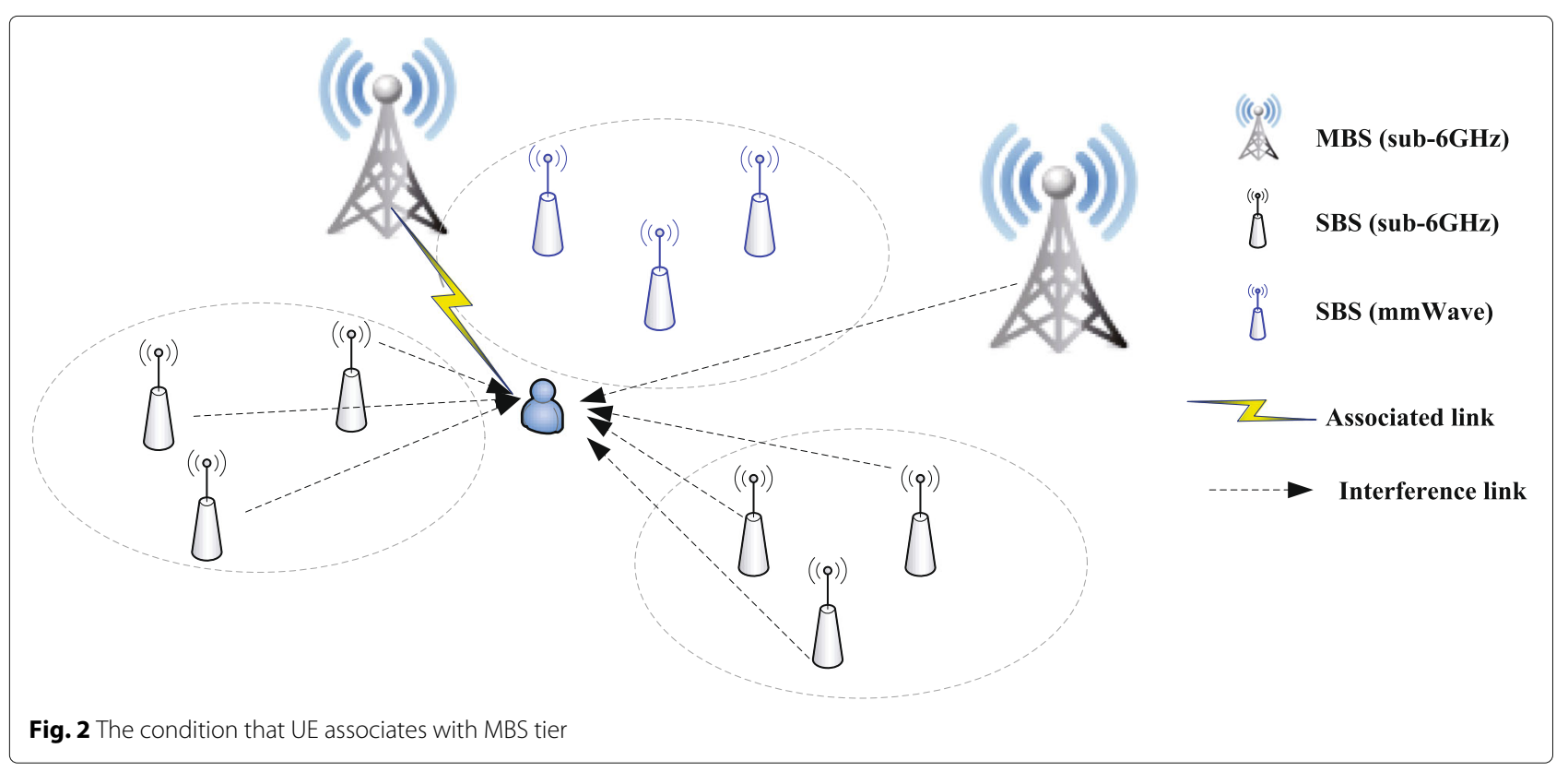




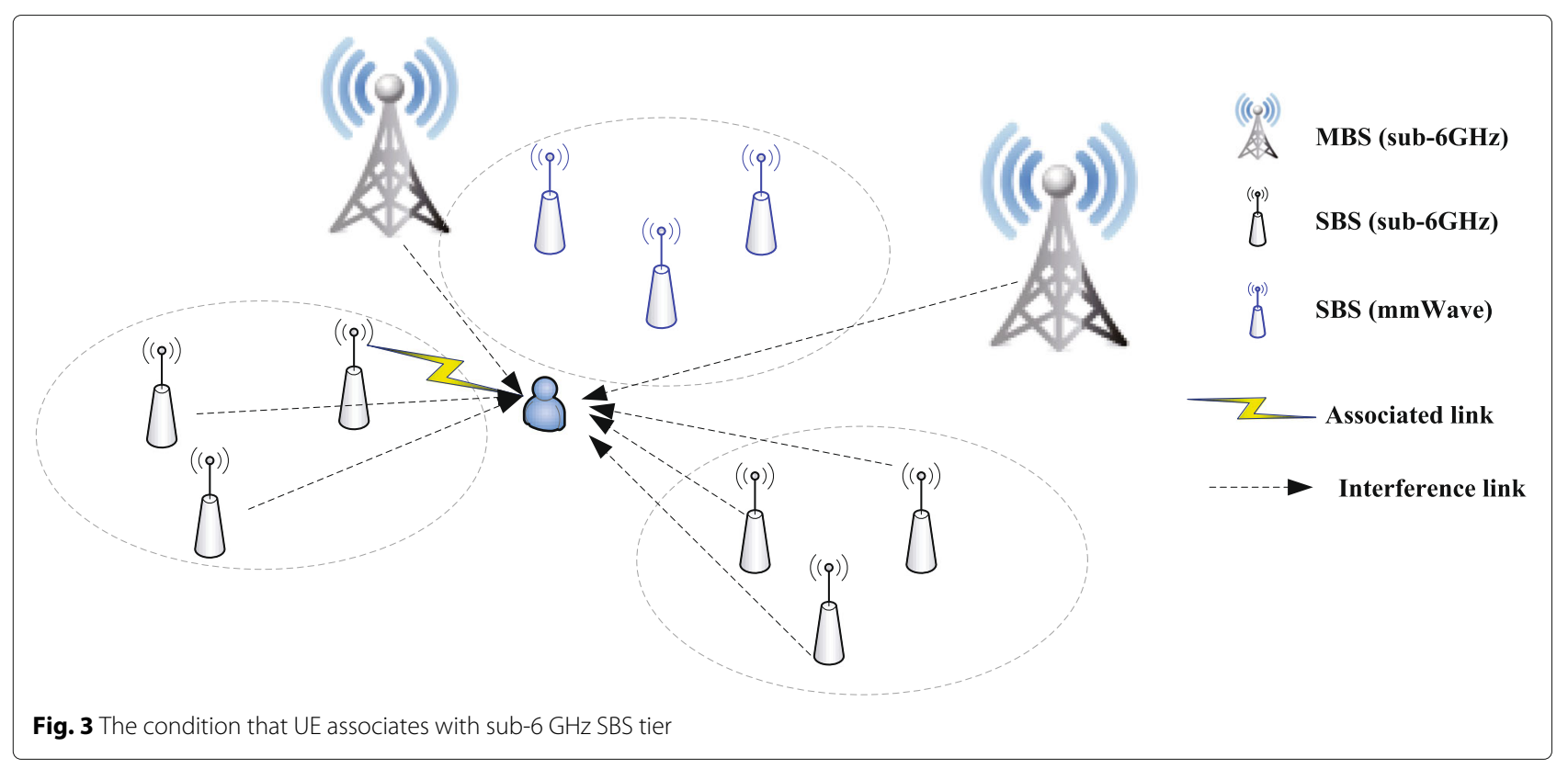

$F_{k i}=\left\{\begin{array}{cc}\frac{\gamma_{T} \beta_{k i}}{\beta_{k k}}, & k \neq i, k \leq N \\ 0, & \text { otherwise }\end{array}\right.$, where $\beta_{k i}=G_{k i} h_{k i}\left\|x_{k i}\right\|^{-\alpha_{k}}$ consists of the antenna gain, channel gain, and path loss from transmitter $k$ to receiver $i$. The elements in $\mathbf{b}$ are auxiliary variables which can be considered as the normalized SINR threshold for every receiver and $\mathbf{b}=$ $\left[\frac{\gamma_{T} N_{0}}{\beta_{11}}, \frac{\gamma_{T} N_{0}}{\beta_{22}}, \ldots, \frac{\gamma_{T} N_{0}}{\beta_{(N+M)(N+M)}}\right]$. The power control vector $\mathbf{P}=\left[P_{1}, P_{2}, \ldots, P_{N}, P_{N+1}, \ldots, P_{N+M}\right]^{T} . P_{\mathrm{mu}}^{\max }$ and $P_{\mathrm{sm}}^{\max }$ are the maximum power of MBS and mmWave SBS, respectively. I is the identity matrix. In this section, we focus on the condition $p_{\mathrm{sm}}=1$, which means that all SBSs operate at the mmWave frequency. Without loss of generality, the results can be extended to the condition $0 \leq p_{\text {sm }}<1$, i.e., the sub- $6 \mathrm{GHz}$ SBSs are included in the optimization.

We can see that the problem in (P1) is non-convex, which is difficult to obtain the optimal solution. Therefore, we decouple this problem into two subproblems, i.e., user association subproblem and power control subproblem. On this basis, we propose a centralized two-stage user association and power control algorithm, denoted by TS algorithm to get the closely local optimum. In the following, we provide the solution to the two subproblems separately.

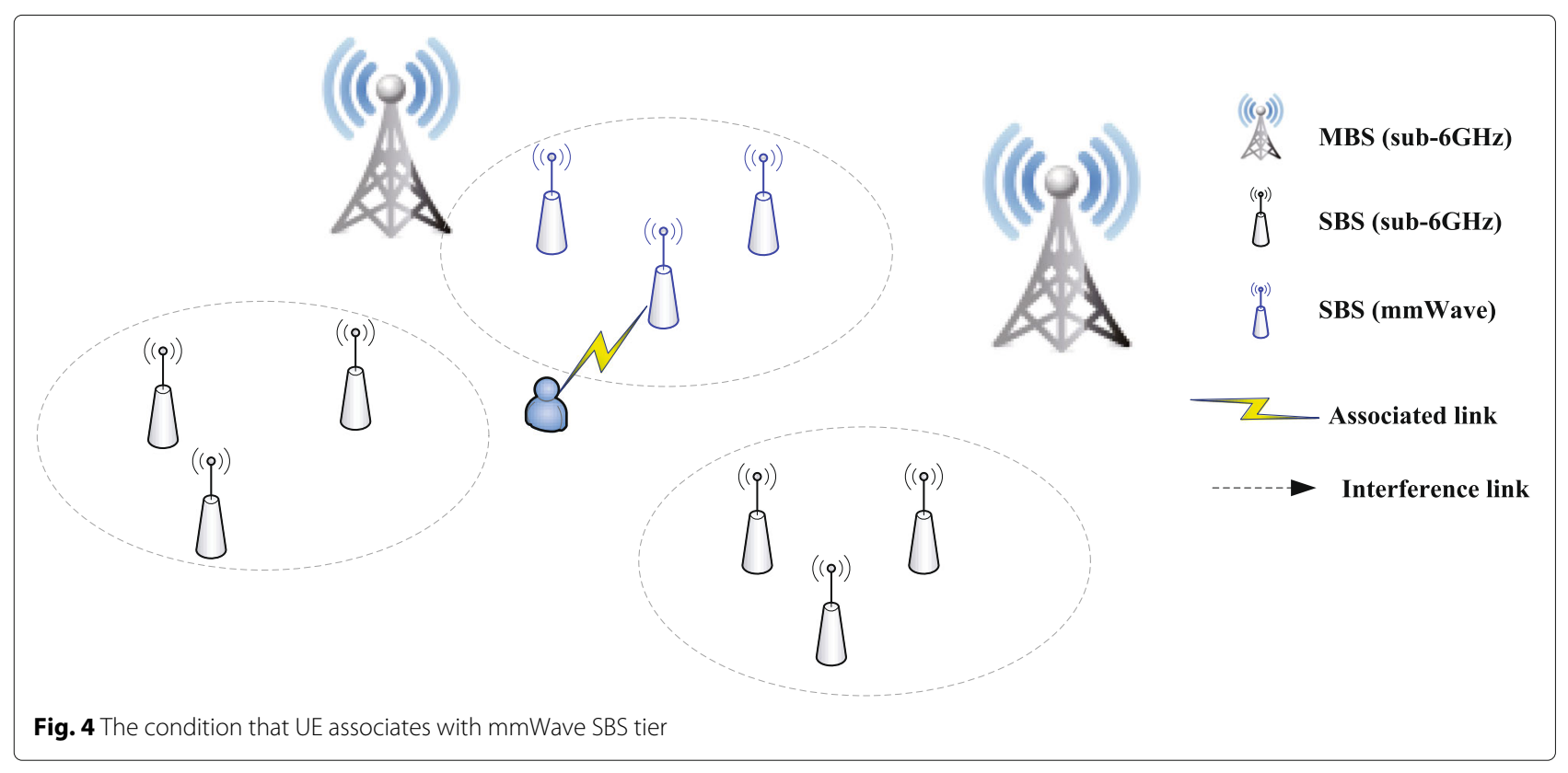




\subsection{The first stage: user association}

The first stage is to find the feasible user association set to satisfy (4) that can be considered as the prerequisite of the next power control. The matrix $\mathbf{F}$ is comprised of non-negative elements and is irreducible because all the links interfere with each other. By the Perron-Frobenious theorem [22, 23], the SINR constraint set in (4) is nonempty if and only if the maximum modulus eigenvalue of $\mathbf{F}$ is smaller than 1, i.e., $\rho(\mathbf{F})<1$. Otherwise, the power control solution is infeasible. The explanation for this conclusion is that the network prefer to support more transmissions, but sometimes it has to drop some links to satisfy SINR constraints. The key idea to solve this problem is to drop communication links successively that cause the maximum sum of the interference in the network until the feasibility conditions are satisfied. It is worthy noting that since the mmWave BSs will not cause the interference due to the high attenuation loss, none of mmWave BSs will be dropped in the first stage.

\subsection{The second stage: power allocation}

After the user association, the original optimization problem (P1) can be reformulated as P2, i.e., a tractable power-constrained sum rate maximization problem.

$$
\begin{aligned}
& \max _{\mathbf{P}} \sum_{i=1}^{M+N^{\prime}} \log \left(1+\operatorname{SINR}_{i}(x)\right), \\
& \text { s.t. } 0 \leq P_{i} \leq P_{\mathrm{mu}}^{\max }, i=1,2, \cdots N^{\prime}, \\
& 0 \leq P_{i} \leq P_{\mathrm{sm}}^{\max }, i=N^{\prime}+1, N^{\prime}+2, \cdots N^{\prime}+M .
\end{aligned}
$$

where $N^{\prime}$ is the number of the updated sub-6 GHz BSs set after the user association stage.

Geometric programming (GP) is a widely adopted method to obtain the optimal solution to the powerconstrained sum rate maximization problem [24] and binary power (BP) can provide the near optimal performance but with low complexity [25]. Therefore, we tackle the power control subproblem by BP. In addition, the optimal mmWave BS transmit power is $P_{i}=P_{\mathrm{sm}}^{\max }, i=N^{\prime}+1$, $N^{\prime}+2, \ldots, N^{\prime}+M$ due to the fact that mmWave link is interference-free.

The details of TS algorithm are describe below.

Centralized two-stage user association and power control algorithm

Initialization: Set gain matrix $\mathbf{F}^{t}$ for $t=0$ according to the global CSI. All cellular and mmWave links are active.

Step 1: Calculate $\rho\left(\mathbf{F}^{t}\right)$. If $\rho\left(\mathbf{F}^{t}\right)<1$, solve the sum rate maximization by $\mathrm{BP}$ to obtain the power allocation method. Then, the algorithm stops. Otherwise, go to step 2 .

Step 2: Calculate $q=\arg \max _{q \in\{1,2, \ldots, N+M\}}\left\|\mathbf{f}_{q}\right\|_{2}$, where $\mathbf{f}_{q}$ is the column of the matrix $\mathbf{F}^{t}$, i.e., $\mathbf{F}^{t}=\left[\mathbf{f}_{1}^{t}, \mathbf{f}_{2}^{t}, \ldots, \mathbf{f}_{N+M}^{t}\right]$.
Step 3: Remove the $q$ th column and row in $\mathbf{F}^{t} . t \leftarrow t+1$. The updated matrix $\mathbf{F}^{t}$ is the reduced matrix. Return to step 1.

\section{Distributed user association analysis of mmWave cellular network}

In the previous section, we propose a centralized twostage user association and power control algorithm. Although centralized algorithm can achieve a good performance, the main challenge is the frequent coordination between transmitters and the high signaling overheads for sharing CSI. In order to solve this problem, we conduct a distributed user association analysis for mmWave cellular network. Specifically, we derive some key performance metrics considering the directivity and blockage effect. On this basis, we propose a distributed distancebased user association method to decide the pattern of BSs (either sub-6 GHz or mmWave pattern).

In order to enable the derivations and the theorems in this section easier to understand. Table 1 lists the symbols and parameters for distributed user association.

For the parameters $k, i, j$, and $l$, they represent $k$-tier, $i$-tier, $j$-tier, and $l$-tier. The following theorem provides the per tier association probability, which is essential for deriving the main results in the sequel.

\subsection{Performance analysis}

In this subsection, we derive the association probability, coverage probability, and the average achievable rate under mmWave cellular network.

Table 1 Symbols and parameters for distributed user association Symbol and parameter Description

$A_{k} \quad$ The probability that a typical user receives the maximum received power from a $k$-tier $B S$.

$r_{k}$

$P_{k}$

The distance from UE to the nearest $k$-tier BS.

$P_{k}$

The transmission power of $k$-tier BS

$\alpha_{k}$

The directivity gain of a $k$-tier link.

$P_{r, k}\left(r_{k}\right)$

The path loss exponent in the $k$-th tier.

The long-term received power from a $k$-tier BS at UE.

$F_{R_{l}}\left(r_{l}\right) \quad$ The cumulative distribution function (cdf) of the distance to the nearest I-tier BS.

$f_{X_{k}}\left(x_{k}\right) \quad$ The probability distribution function (pdf) of $k$-tier associated distance $x_{k}$.

$\operatorname{SINR}_{k}(x) \quad$ The received SINR at the typical user (at origin o) conditioned on the associated $k$-tier BS at $x$.

$\Re_{k} \quad$ The average achievable rate on the condition that user is associated with $k$-tier.

$\Re$

The average achievable rate.

$\varphi_{\mathrm{sm}}$

The sm-tier coverage probability conditioned on the associated distance.

$\varphi_{\mathrm{mu}}$

The mu-tier coverage probability conditioned on the associated distance. 
The following theorem provides the per tier association probability, which is essential for deriving the main results in the sequel.

Theorem 1 The association probability in the kth tier is

$$
A_{k}=\left\{\begin{array}{c}
\mathbb{E}_{r_{k}}\left[\operatorname { e x p } ( - \pi \lambda _ { j } ( \frac { P _ { j } } { P _ { k } } ) ^ { \frac { 2 } { \alpha _ { s } } } r _ { k } ^ { 2 } ) \left(\frac{\omega}{\pi}\left(1-\mathcal{E}_{Y_{l}}\left(\left(\frac{P_{l} G_{\max }}{P_{k}}\right) r_{k}^{\alpha_{s}}\right)\right)\right.\right. \\
\left.\left.+\frac{\pi-\omega}{\pi}\left(1-\mathcal{E}_{Y_{l}}\left(\left(\frac{P_{l} G_{\min }}{P_{k}}\right) r_{k}^{\alpha_{s}}\right)\right)\right)\right], \\
\quad j, k \in\{\mathrm{mu}, \mathrm{su}\}, j \neq k, l=\mathrm{sm} \\
\mathbb{E}_{r_{k}}\left[\frac{\omega}{\pi} \exp \left(-\pi \sum_{j \in\{\mathrm{mu}, \mathrm{su}\}} \lambda_{j}\left(\frac{P_{j}}{P_{k} G_{\max }}\right)^{\frac{2}{\alpha_{s}}} r_{k}^{2}\right)\right. \\
\left.+\frac{\pi-\omega}{\pi} \exp \left(-\pi \sum_{j \in\{\mathrm{mu}, \mathrm{su}\}} \lambda_{j}\left(\frac{P_{j}}{P_{k} G_{\min }}\right)^{\frac{2}{\alpha_{s}}} r_{k}^{2}\right)\right], \\
\left.k=\mathrm{sm}{ }^{-\pi}\right)
\end{array}\right.
$$

where $\mathcal{E}_{Y_{l}}(y)$ is expressed as

$$
\begin{aligned}
& \mathcal{E}_{Y_{l}}(y)
\end{aligned}
$$

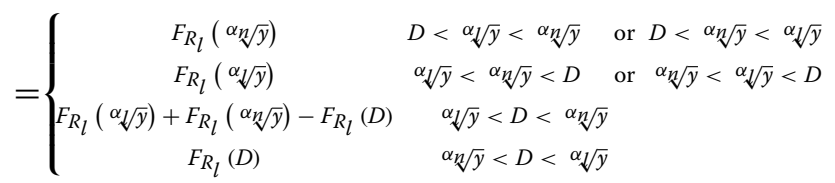

where $F_{R_{l}}\left(r_{l}\right)=1-\exp \left(-\pi \lambda_{l} r_{l}^{2}\right)$ is the cumulative distribution function (cdf) of the distance of the nearest l-tier $B S$.

\section{Proof See Appendix A.}

From Theorem 1, we further derive the the distance between a user and its serving BS, of which the premise is that the user is associated with the $k$ th tier.

Corollary 1 The probability distribution function (pdf) of $k$-tier associated distance

$$
f_{X_{k}}\left(x_{k}\right)=-\frac{\partial \operatorname{Pr}\left[r_{k}>x_{k}, P_{r, k}>\max _{j, j \neq k} P_{r . j}\right]}{A_{k} \partial x_{k}}
$$

where $\operatorname{Pr}\left[r_{k}>x_{k}, P_{r, k}>\max _{j, j \neq k} P_{r, j}\right]$ is obtained through the method to calculate $A_{k}$, in which the only difference is the integral interval over $r_{k}$ is $x_{k}$ to infinity instead of zero to infinity.

Depending on the association probability and the pdf of the associated distance, we further obtain the coverage probability and average achievable rate, which are the two important indicators to evaluate the network performance.

In this paper, a UE is said to be in coverage if it is able to connect to at least one BS with SINR above its threshold.
Theorem 2 The coverage probability is

$$
\begin{aligned}
& \operatorname{Pr}\left(\bigcup_{k \in\{m u, s u, s m\}} \operatorname{SINR}_{k}\left(x_{k}\right)>\gamma_{k}\right)=\mathbb{E}_{x_{k}}\left[\frac{\omega}{\pi} \exp \left(-\frac{\gamma_{k} N_{0}}{P_{k} G_{\max } x_{k}^{-\alpha_{k}}}\right)\right. \\
& \left.+\frac{\pi-\omega}{\pi} \exp \left(-\frac{\gamma_{k} N_{0}}{P_{k} G_{\min } x_{k}^{-\alpha_{k}}}\right)\right]+\sum_{k \in\{m u, s u\}} \mathbb{E}_{x_{k}}\left[\exp \left(-\frac{\gamma_{k} \sum_{z \in \Phi_{j}} P_{j} h_{z} z^{-\alpha_{S}}}{P_{k} x_{k}^{-\alpha_{s}}}\right)\right] \\
& \mathbb{E}_{x_{k}}^{!}\left[\exp \left(-\frac{\gamma_{k} \sum_{z \in \Phi_{j}} h_{z} z^{-\alpha_{s}}}{x_{k}^{-\alpha_{s}}}\right)\right]
\end{aligned}
$$

where the first term can be calculated by averaging over $x_{k}$, and the pdf of $x_{k}$ is given by Corollary 1. $\mathbb{E}_{x_{k}}\left[\exp \left(-\frac{\gamma_{k} \sum_{z \in \Phi_{j}} P_{j} h_{z} z^{-\alpha_{s}}}{P_{k} x_{k}^{-\alpha_{s}}}\right)\right]$ and $\mathbb{E}_{x_{k}}^{!}\left[\exp \left(-\frac{\gamma_{k} \sum_{z \in \Phi_{j}} h_{z} z^{-\alpha_{S}}}{x_{k}^{-\alpha_{S}}}\right)\right]$ have been derived in [19].

Proof See Appendix B.

Last, we derive the average achievable rate of mmWave cellular network. The average achievable rate $\mathfrak{R}$ can be derived by the method to analyze the coverage probability in Appendix B.

Theorem 3 The average achievable rate is

$$
\begin{aligned}
\Re= & \sum_{k \in\{m u, s u, s m\}} A_{k} \Re_{k}=\sum_{k \in\{m u, s u, s m\}} \\
& A_{k} \mathbb{E}_{x_{k}}\left[\int_{0}^{\infty} \operatorname{Pr}\left(\operatorname{SINR}_{k}\left(x_{k}\right)>e^{t}-1\right) d t\right]
\end{aligned}
$$

where $\Re_{k}$ is the average achievable rate on the condition that user is associated with $k$-tier and $\operatorname{Pr}\left(\operatorname{SINR}_{k}\left(x_{k}\right)>e^{t}-1\right)$ can be easily deduced according to (25) and (26) in Appendix B.

\subsection{Distributed distance-based user association method}

On the basis of the expression of coverage probability, together with the known associated distance, we propose a simple method to judge which pattern of BS (sub- $6 \mathrm{GHz}$ or mmWave) are preferred by comparing the conditional coverage probabilities of sub-6 GHz BS and mmWave BSs. In order to make the problem mathematically trackable, we set $p_{\mathrm{sm}}=1$ in the following analysis.

The sm-tier coverage probability conditioned on the associated distance is

$$
\begin{aligned}
\varphi_{\mathrm{sm}}= & \frac{\omega}{\pi} \exp \left(-\frac{\gamma_{\mathrm{sm}} N_{0}}{P_{\mathrm{sm}} G_{\mathrm{max}} x_{\mathrm{sm}}^{-\alpha_{\mathrm{sm}}}}\right) \\
& +\frac{\pi-\omega}{\pi} \exp \left(-\frac{\gamma_{\mathrm{sm}} N_{0}}{P_{\mathrm{sm}} G_{\mathrm{min}} x_{\mathrm{sm}}^{-\alpha_{\mathrm{sm}}}}\right)
\end{aligned}
$$

The mu-tier coverage probability conditioned on the associated distance is [16] 


$$
\begin{aligned}
\varphi_{\mathrm{mu}}= & \exp \left\{-2 \pi \lambda_{\mathrm{mu}} x_{\mathrm{mu}}^{2} \gamma_{\mathrm{mu}}^{\frac{2}{\alpha_{s}}}\left[B\left(1 ; \frac{2}{\alpha_{s}}, 1-\frac{2}{\alpha_{s}}\right)\right.\right. \\
& \left.\left.-B\left(\frac{1}{1+\gamma_{\mathrm{mu}}} ; \frac{2}{\alpha_{s}}, 1-\frac{2}{\alpha_{s}}\right)\right]\right\}
\end{aligned}
$$

where $B(x ; y, z)$ is the incomplete beta function.

The user association decision is determined by the coverage probability conditioned on the associated distance, that is, the user will associate to the nearest mmWave BS if $\varphi_{\mathrm{sm}} \geq \varphi_{\mathrm{mu}}$, otherwise associates to the nearest MBS.

Remark 1 According to Jensen inequality, [26] $a_{1} \exp \left(-b_{1} x^{\alpha_{\mathrm{sm}}}\right)+a_{2} \exp \left(-b_{2} x^{\alpha_{\mathrm{sm}}}\right) \geq \exp \left(-\left(a_{1} b_{1}+\right.\right.$ $\left.\left.a_{2} b_{2}\right) x^{\alpha_{\mathrm{sm}}}\right)$, the sufficient condition for the inequality $\varphi_{\mathrm{sm}} \geq \varphi_{\mathrm{mu}}$ is $\exp \left(-\left(a_{1} b_{1}+a_{2} b_{2}\right) x^{\alpha_{\mathrm{sm}}}\right) \geq \exp \left(-c x^{2}\right)$. Therefore, the user will associate with nearest mmWave BS if $D \leq x \leq\left(\frac{c}{a_{1} b_{1}+a_{2} b_{2}}\right)^{\frac{1}{\alpha_{n}-2}}$ or $x<\min \left\{D,\left(\frac{c}{a_{1} b_{1}+a_{2} b_{2}}\right)^{\frac{1}{\alpha_{l}-2}}\right\}$.

\section{Results and discussion}

In this section, we present numerical and simulation results on association probability, coverage probability, and average rate for mmWave cellular network and then evaluates the performance of the proposed centralized TS algorithm. The simulation parameters are listed in Table 2.

We start by looking into the association probabilities and the different factors that affect the probability. Figure 5 illustrates the association probability of sub$6 \mathrm{GHz}$ MBS, sub-6 GHz SBS, and mmWave SBS against the mmWave probability $p_{\mathrm{sm}}$. Figure 5 shows that the simulation results match the analytic results well, which validates our analysis. It is also seen in Fig. 1 that more UEs associate with mmWave BS with the growth of $p_{\mathrm{sm}}$, which is in agreement with our intuition. Moreover, it can be seen that MBSs still undertake more UEs due to higher transmission power.

Table 2 Simulation parameters

\begin{tabular}{ll}
\hline Parameters & Value \\
\hline Side length of simulation area & $L=1500 \mathrm{~m}$ \\
MBS density & $\lambda_{m}=5 / \pi L^{2}$ \\
SBS parent density & $\lambda_{p}=2 \lambda_{m}$ \\
The number of SBS in a cluster & $c=[4: 10]$ \\
The probability of mmWave BS among SBSs & $P_{\text {sm }}=[0: 0.1: 1]$ \\
Cluster range of SBS & $R=[50: 100: 750]$ \\
Transmit power of MBS & $P_{\mathrm{m}}=46 \mathrm{dBm}=39 \mathrm{~W}[10]$ \\
Transmit power of SBS & $P_{\mathrm{su}}=P_{\mathrm{sm}}=30 \mathrm{dBm}=1 \mathrm{~W}[10]$ \\
SINR threshold & $\gamma_{T}=[-10: 2: 10] \mathrm{dB}$ \\
Antenna gain & $\omega=5, \mathrm{G}_{\mathrm{max}}=18 \mathrm{dBi}=63, \mathrm{G}_{\mathrm{min}}=-2 \mathrm{dBi}=0.63[10]$ \\
Path loss & $\alpha_{s}=3, \alpha=2, \alpha_{n}=4[27]$ \\
Small-scale fading & $h \sim \exp (1)$ \\
mmWave bandwidth & $W_{s}=1 \mathrm{GHz}[9]$ \\
Noise power of mmWave & $-174 \mathrm{dBm} / \mathrm{Hz}+10 \log \left(W_{s}\right)+10 \mathrm{~dB}[9]$ \\
\hline
\end{tabular}

Figure 6 shows that coverage probability decreases with the larger $c$ value whereas the gap between different $c$ diminishes with the increasing mmWave probability $p_{\mathrm{sm}}$. That is because larger $c$ value under low $p_{\text {sm }}$ results in more sub-6 GHz SBSs that cause more interference. However, the effect of $c$ on coverage probability becomes weaker with the increasing $p_{\text {sm }}$ due to the fact that more SBSs conduct in interference-free mmWave pattern with the increase of $p_{\mathrm{sm}}$. The extreme case is that the gap disappears for $p_{\mathrm{sm}}=1$, as is shown in Fig. 2. Thus, the results in Fig. 2 demonstrates that mmWave links can contribute to coverage probability.

Similarly, the higher achievable average rate is along with the larger $p_{\text {sm }}$ in Fig. 7 . It is worth noting that a cross point appears with the increasing $p_{\mathrm{sm}}$ under different $c$. That means less sub- $6 \mathrm{GHz}$ SBSs (i.e., lower interference) or more mmWave SBSs (i.e., better channel state) can achieve higher achievable average rate.

From Fig. 8, we can see that the cluster radius $R$ has no strong effect on UE association when SBS density is small, which further verifies that a PCP can be approximated as a PPP under small value $c$. Moreover, it is noticeable that mmWave support highest data rate even under the least number of associated UEs by comparing Figs. 8 and 9 . Therefore, mmWave communications outperforms sub$6 \mathrm{GHz}$ communications on rate delivery for high directivity and interference-free transmission environment. It can be seen in Fig. 10 that the coverage probability grows in accordance with the increasing cluster radius whereas it tends to keep stable for small values of $c$. Higher $c$ value results in stronger non-uniform distribution, which can implicitly confirm that non-uniformity can reduce coverage area.

This finding obtained in Fig. 11 is in agreement with the published paper [6] that the coverage probability 


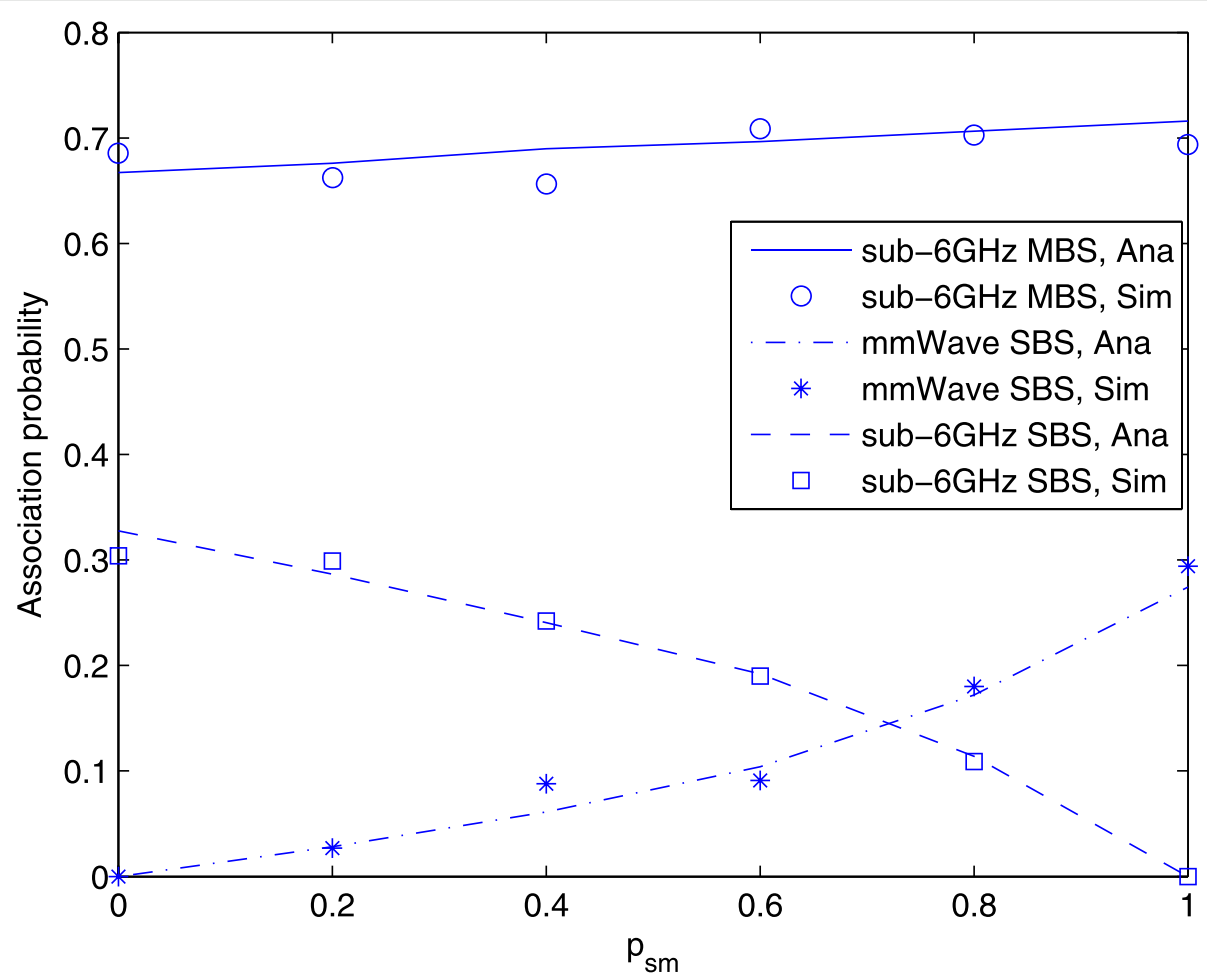

Fig. 5 Association probability vs. the mmWave probability $p_{\mathrm{sm}}$

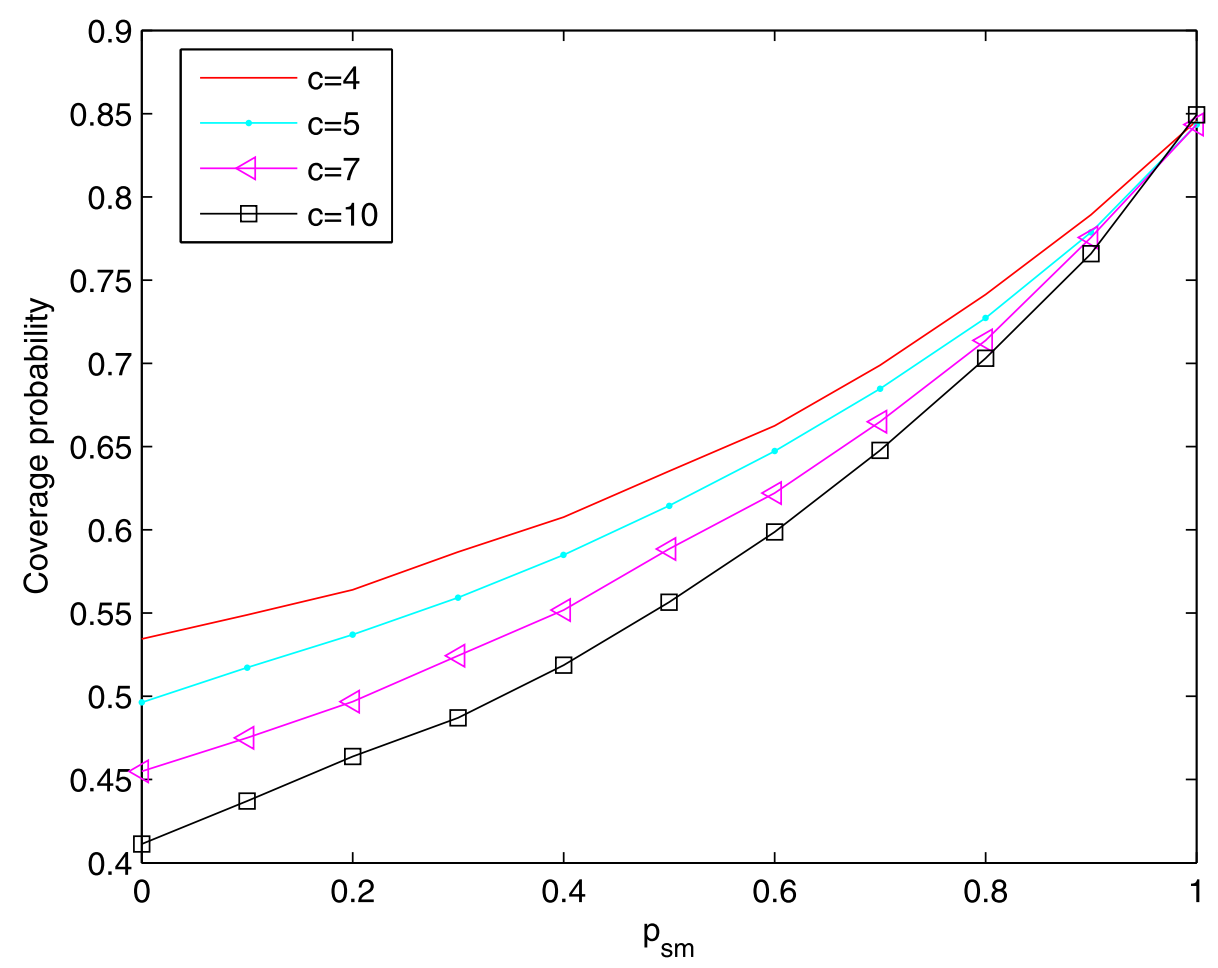

Fig. 6 Coverage probability vs. the mmWave probability $p_{s m}$ 


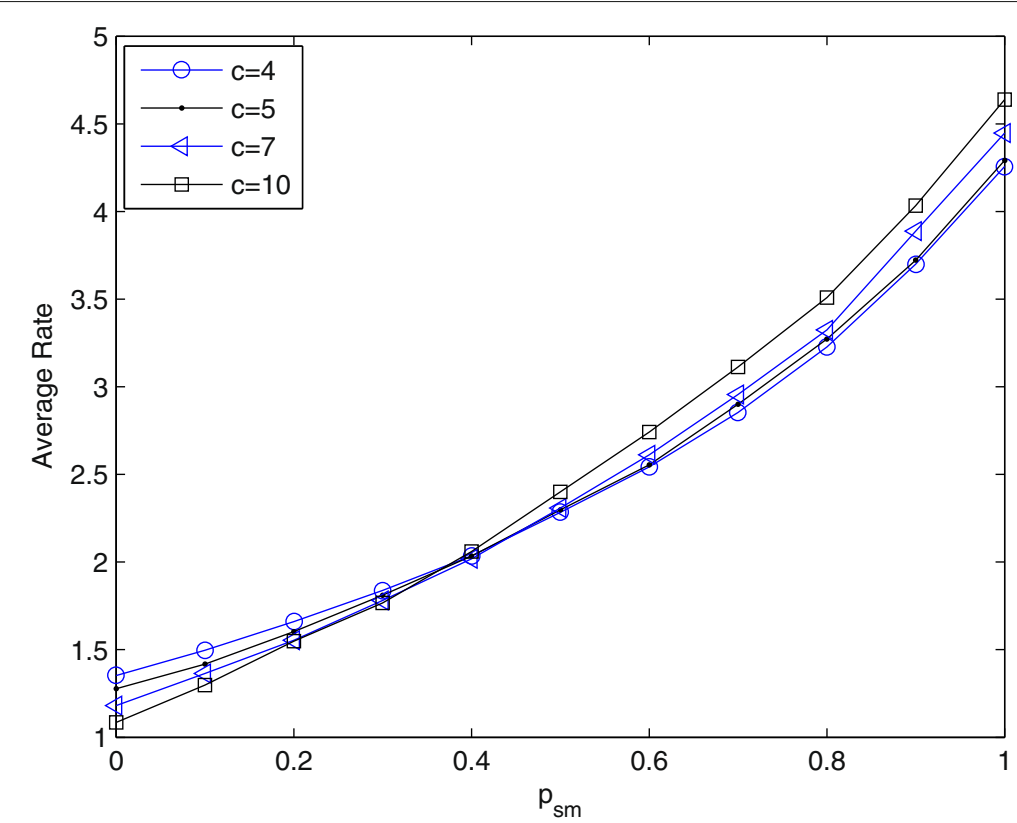

Fig. 7 Average achievable rate vs. the mmWave probability $p_{\mathrm{sm}}$

decreases with the growing SINR threshold. Also, it illustrates that more mmWave links are able to enhance the coverage probability. This is mainly due to interference-free transmission environment under mmWave communications.
As expected, the sum rate decreases with the increasing SINR threshold in Fig. 8. Meanwhile, we compare the performance of the proposed centralized TS algorithm (using BP) and geometric programming (GP) algorithm where the performance of GP is commonly adopted as a

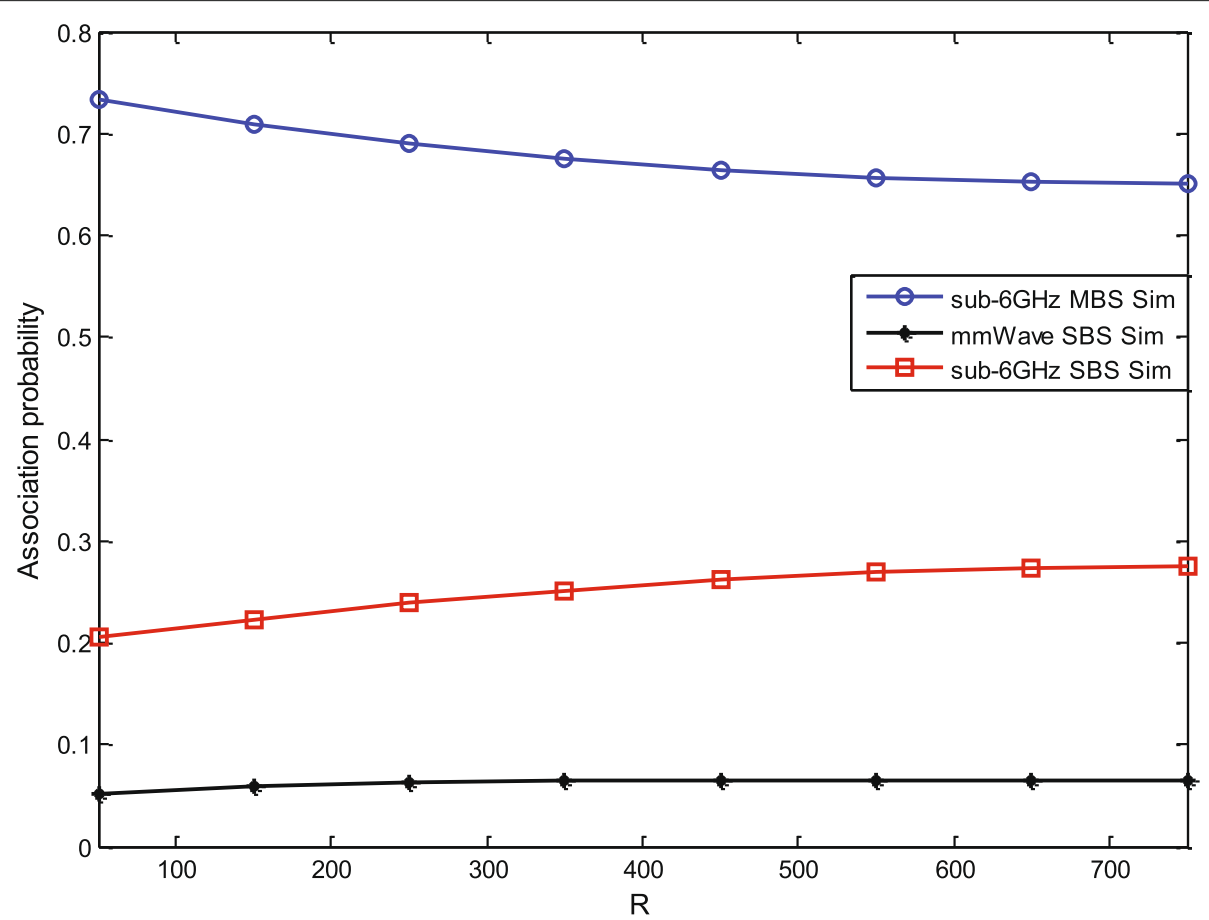

Fig. 8 Association probability vs. the cluster radius $R$ 


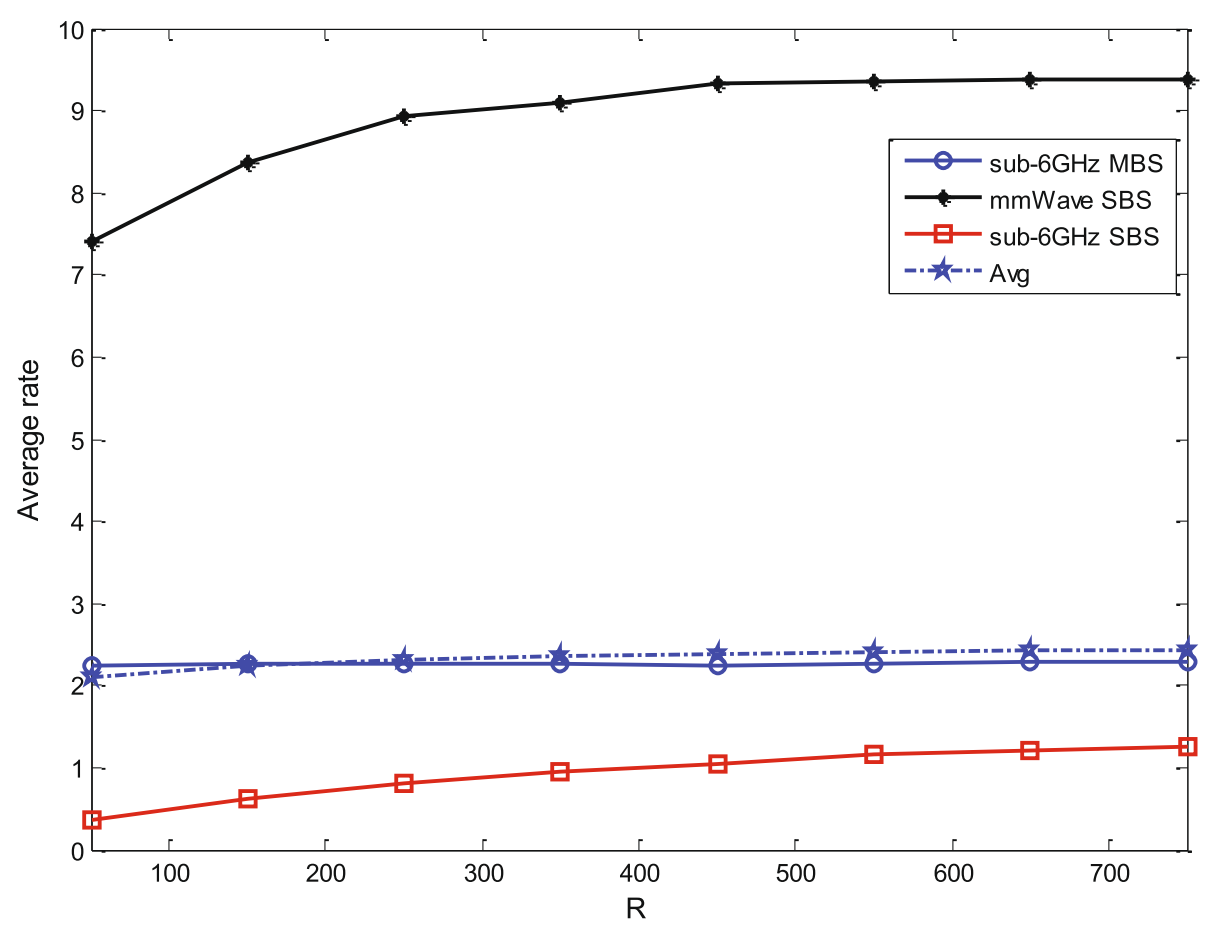

Fig. 9 Average achievable rate vs. the cluster radius $R$

benchmark. The observation in Fig. 12 is that TS algorithm is always approximate to the GP for $c=1$. Although the gap between GP and BP becomes obvious with the larger $c, \mathrm{BP}$ is near to the optimal results under low SINR. The results demonstrate near-optimality of BP under low SINR constraint. Moreover, the complexity of the BP-based transmitter design is reduced since only a two-level power control is required, which is a potential in the design and analysis of wireless networks.

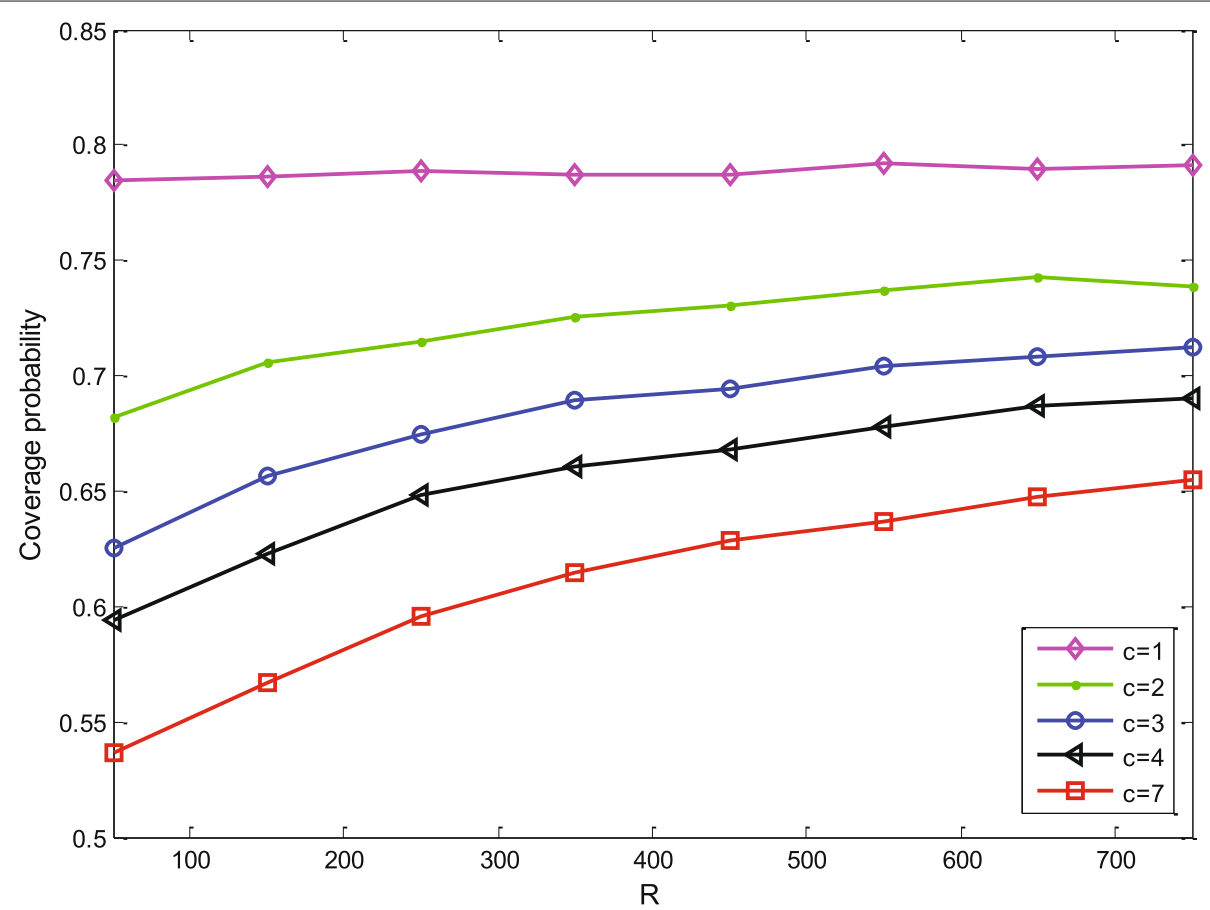

Fig. 10 Coverage probability vs. the cluster radius $R$ 


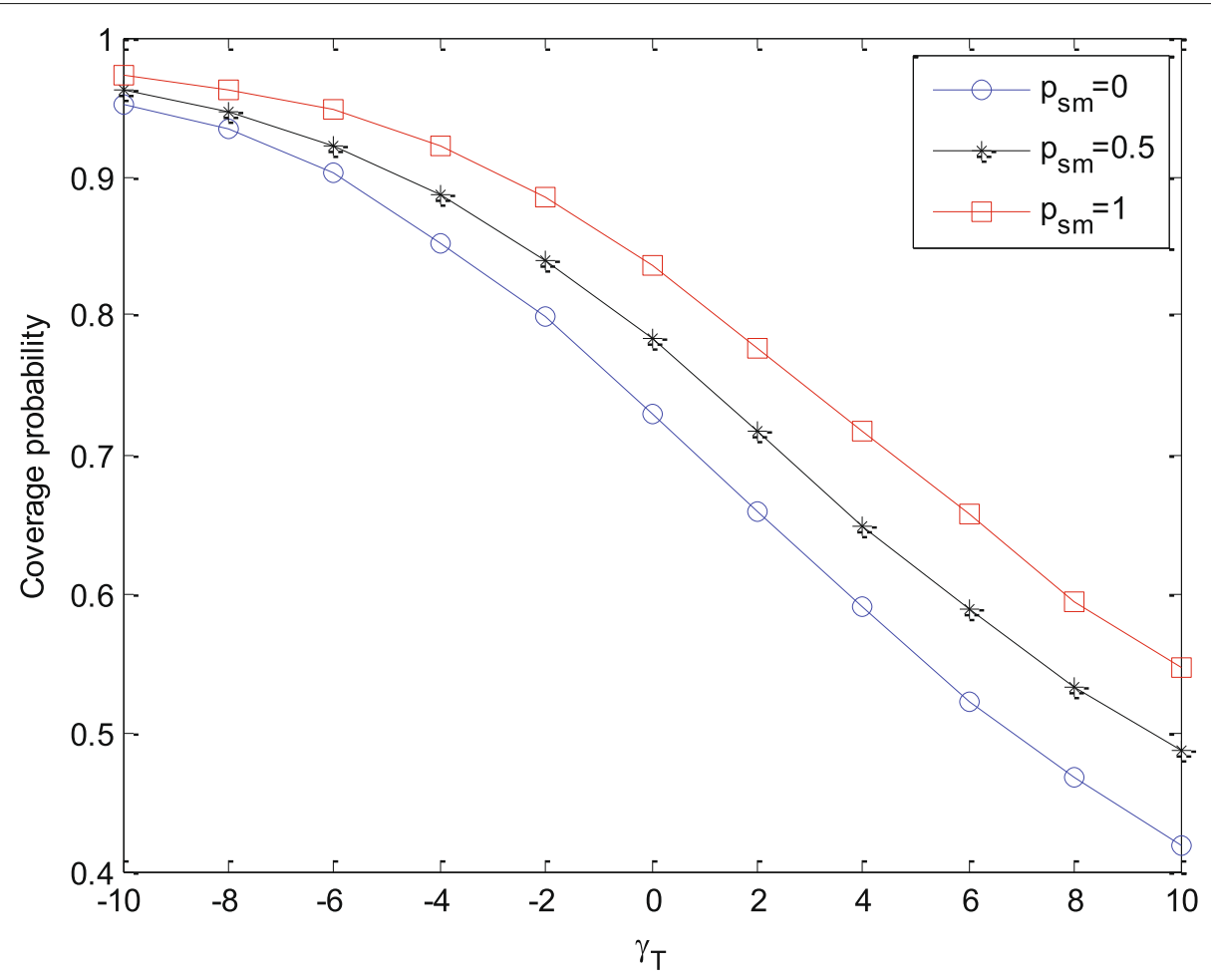

Fig. 11 Coverage probability vs. SINR threshold

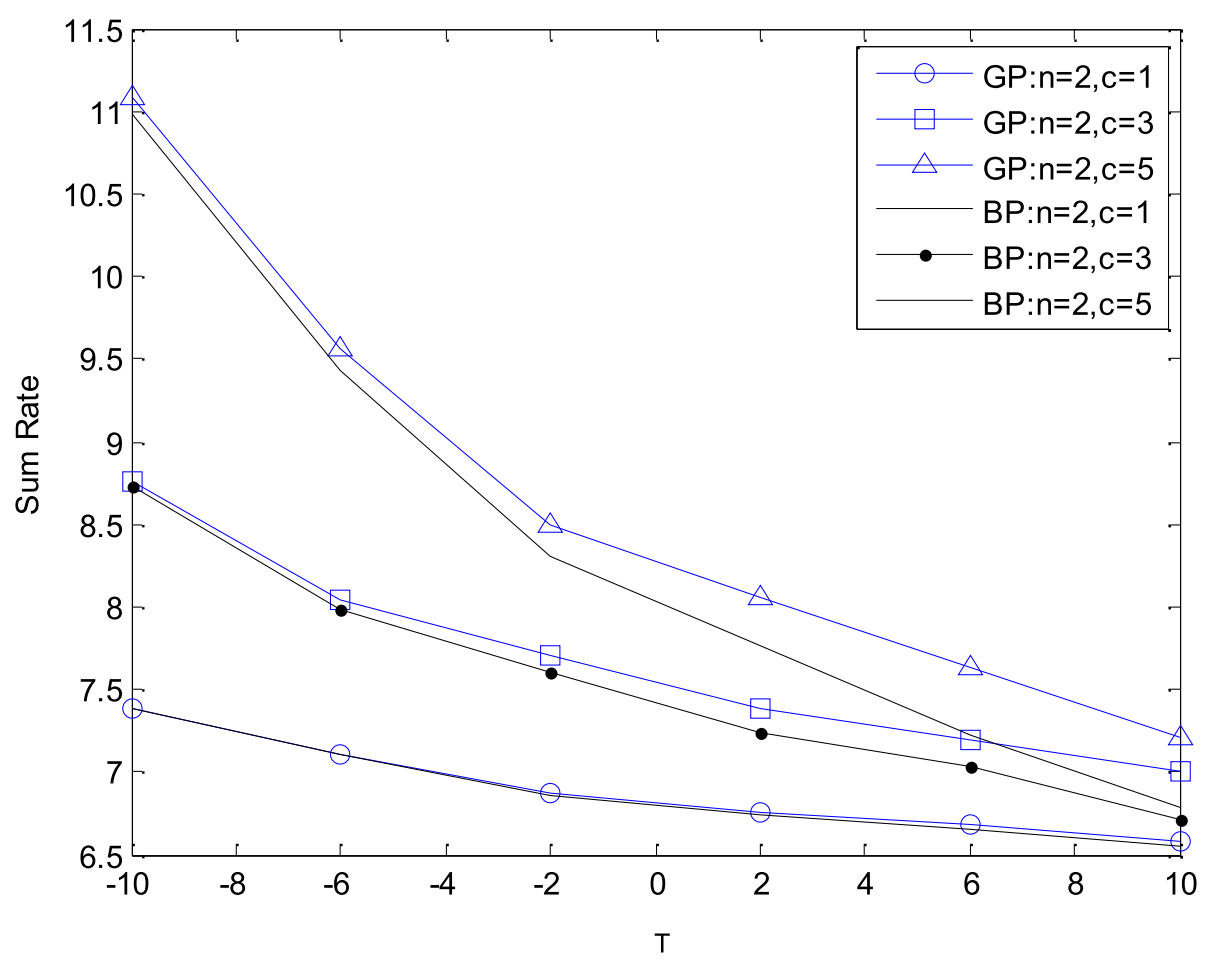

Fig. 12 Sum rate of the centralized algorithms vs. SINR threshold 


\section{Conclusion}

This paper proposes a realistic multi-tier mmWave cellular framework where sub- $6 \mathrm{GHz}$ MBS deployment is assumed as a PPP, and SBS operating on either mmWave or sub- $6 \mathrm{GHz}$ follows PCP model. In this system, we propose both centralized and distributed user association algorithms. For the centralized two-step algorithm, we aim to maximize the sum rate while satisfying QoS and power consumption constraints based on eigenvalue analysis. Then, we derive the association probability, the coverage probability, and the average achievable rate, incorporating directivity and blockage effect, by stochastic geometry. On this basis, the distributed user association algorithm is proposed. The simulation results demonstrate the accuracy of our theoretical analysis and also reveal that the effect of some parameters on the network performance. In addition, the proposed centralized algorithm can achieve near-optimal sum rate with a low complexity under nondense scenario.

\section{Appendix}

\section{A. Proof of Theorem 1}

The associated probability in the $k$-tier is given by

$$
\begin{aligned}
A_{k} & =\operatorname{Pr}\left[P_{r, k}>\max _{j, j \neq k} P_{r, j}\right]=\mathbb{E}_{r_{k}, \mathbf{G}}\left[\operatorname{Pr}\left(P_{k} G_{k} r_{k}^{-\alpha_{k}}>\max _{j, j \neq k} P_{j} G_{j} r_{j}^{-\alpha_{j}}\right)\right] \\
& \stackrel{(a)}{=} \mathbb{E}_{r_{k}, \mathbf{G}}\left[\prod_{j \in\{\text { mu,su,sm }, j \neq k} \operatorname{Pr}\left(P_{k} G_{k} r_{k}^{-\alpha_{k}}>P_{j} G_{j} r_{j}^{-\alpha_{j}}\right)\right]
\end{aligned}
$$

where $a$ is given from the independence of PPP. The pdf of $r_{k}$ is given by [16]. $\mathbf{G}$ is a vector involving the directivity gains of all links.

First we calculate $\operatorname{Pr}\left(P_{k} G_{k} r_{k}^{-\alpha_{k}}>P_{j} G_{j} r_{j}^{-\alpha_{j}}\right)$

$$
\begin{aligned}
& \text { 1. If } k, j \in\{\mathrm{mu}, \mathrm{su}\} \text { and } j \neq k \\
& \begin{aligned}
\operatorname{Pr}\left(P_{k} G_{k} r_{k}^{-\alpha_{k}}>P_{j} G_{j} r_{j}^{-\alpha_{j}}\right) & =\operatorname{Pr}\left(r_{j}>\left(\frac{P_{j}}{P_{k}}\right)^{\frac{1}{\alpha_{s}}} r_{k}\right) \\
& =\exp \left(-\pi \lambda_{j}\left(\frac{P_{j}}{P_{k}}\right)^{\frac{2}{\alpha_{s}}} r_{k}^{2}\right)
\end{aligned}
\end{aligned}
$$

2. If $k \in\{\mathrm{mu}, \mathrm{su}\}$ and $j=\mathrm{sm}$

$$
\begin{gathered}
\operatorname{Pr}\left(P_{k} G_{k} r_{k}^{-\alpha_{k}}>P_{j} G_{j} r_{j}^{-\alpha_{j}}\right)=\operatorname{Pr}\left(r_{j}^{\alpha_{j}}>\left(\frac{P_{j} G_{j}}{P_{k} G_{k}}\right) r_{k}^{\alpha_{k}}\right) \\
=1-\int_{0}^{\left(\frac{P_{j} G_{j}}{P_{k}}\right) r_{k}^{\alpha_{s}}} \ell_{Y_{j}}(y) d y=1-\mathcal{E}_{Y_{j}}\left(\left(\frac{P_{j} G_{j}}{P_{k}}\right) r_{k}^{\alpha_{s}}\right)
\end{gathered}
$$

where the variable $Y_{j}=r_{j}^{\alpha_{j}} \cdot \ell_{Y_{j}}(y)$ and $\mathcal{E}_{Y_{j}}(y)$ are the pdf and cdf of variable $Y_{j}$, respectively. The cdf of variable $Y_{j}$ is given by

$$
\mathcal{E}_{Y_{j}}(y)=\operatorname{Pr}\left(Y_{j} \leq y\right)=\operatorname{Pr}\left(r_{j} \leq \sqrt[\alpha_{j}]{y}\right)
$$

where the last term in (19) can be calculated based on the pdf of distance between user and the nearest $j$-tier BS. Therefore, $\mathcal{E}_{Y_{j}}(y)$ is expressed as

$$
\begin{aligned}
& \mathcal{E}_{Y_{j}}(y)=
\end{aligned}
$$

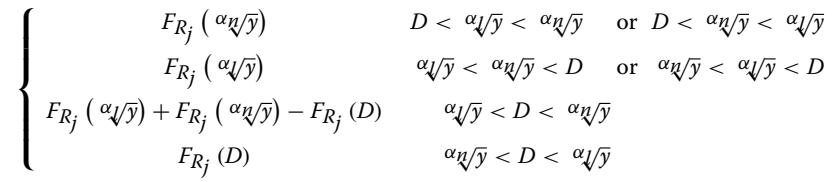

where $F_{R_{j}}\left(r_{j}\right)=1-\exp \left(-\pi \lambda_{j} r_{j}^{2}\right)$ is the cdf of the distance of the nearest $j$-tier BS.

$$
\text { 3. If } k=\operatorname{sm} \text { and } j \in\{\mathrm{mu}, \mathrm{su}\}
$$

$$
\begin{aligned}
& \operatorname{Pr}\left(P_{k} G_{k} r_{k}^{-\alpha_{k}}>P_{j} G_{j} r_{j}^{-\alpha_{j}}\right)= \\
& \left\{\begin{array}{l}
\operatorname{Pr}\left(r_{j}>\left(\frac{P_{j} G_{j}}{P_{k} G_{k}}\right)^{\frac{1}{\alpha_{s}}} r_{k}^{\frac{\alpha_{l}}{\alpha_{s}}}\right)=\exp \left(-\pi \lambda_{j}\left(\frac{P_{j}}{P_{k} G_{k}}\right)^{\frac{2}{\alpha_{s}}} r_{k}^{\frac{2 \alpha_{l}}{\alpha_{s}}}\right) r_{k} \leq D \\
\operatorname{Pr}\left(r_{j}>\left(\frac{P_{j} G_{j}}{P_{k} G_{k}}\right)^{\frac{1}{\alpha_{s}}} r_{k}^{\frac{\alpha_{n}}{\alpha_{s}}}\right)=\exp \left(-\pi \lambda_{j}\left(\frac{P_{j}}{P_{k} G_{k}}\right)^{\frac{2}{\alpha_{s}}} r_{k}^{\frac{2 \alpha_{n}}{\alpha_{s}}}\right) r_{k}>D
\end{array}\right.
\end{aligned}
$$

Thus, the $k$-tier associated probability is

$$
\begin{gathered}
A_{k}=\left\{\begin{array}{c}
\mathbb{E}_{r_{k}, \mathbf{G}}\left[\exp \left(-\pi \lambda_{j}\left(\frac{P_{j}}{P_{k}}\right)^{\frac{2}{\alpha_{s}}} r_{k}^{2}\right)\left(1-\mathcal{E}_{Y_{l}}\left(\left(\frac{P_{l} G_{l}}{P_{k}}\right) r_{k}^{\alpha_{s}}\right)\right)\right], j, k \in\{\mathrm{mu}, \mathrm{su}\}, j \neq k, l=\mathrm{sm} \\
\mathbb{E}_{r_{k}, \mathbf{G}}\left[\exp \left(-\pi \sum_{j \in\{\mathrm{mu}, \mathrm{su}\}} \lambda_{j}\left(\frac{P_{j}}{P_{k} G_{k}}\right)^{\frac{2}{\alpha_{s}}} r_{k}^{2}\right)\right] \quad k=\mathrm{sm}
\end{array}\right. \\
\stackrel{(a)}{=}\left\{\begin{array}{r}
\mathbb{E}_{r_{k}}\left[\exp \left(-\pi \lambda_{j}\left(\frac{P_{j}}{P_{k}}\right)^{\frac{2}{\alpha_{s}}} r_{k}^{2}\right)\left(\frac{\omega}{\pi}\left(1-\mathcal{E}_{Y_{l}}\left(\left(\frac{P_{l} G_{\max }}{P_{k}}\right) r_{k}^{\alpha_{s}}\right)\right)+\frac{\pi-\omega}{\pi}\left(1-\mathcal{E}_{Y_{l}}\left(\left(\frac{P_{l} G_{\min }}{P_{k}}\right) r_{k}^{\alpha_{s}}\right)\right)\right)\right], \\
j, k \in\{\mathrm{mu}, \mathrm{su}\}, j \neq k, l=\mathrm{sm}
\end{array}\right. \\
\mathbb{E}_{r_{k}}\left[\frac{\omega}{\pi} \exp \left(-\pi \sum_{j \in\{\mathrm{mu}, \mathrm{su}\}} \lambda_{j}\left(\frac{P_{j}}{P_{k} G_{\max }}\right)^{\frac{2}{\alpha_{s}}} r_{k}^{2}\right)+\frac{\pi-\omega}{\pi} \exp \left(\begin{array}{c}
\left.\left.\sum_{j \in\{\mathrm{mu}, \mathrm{su}\}} \lambda_{j}\left(\frac{P_{j}}{P_{k} G_{\min }}\right)^{\frac{2}{\alpha_{s}}} r_{k}^{2}\right)\right], \\
k=\mathrm{sm}
\end{array}\right.\right.
\end{gathered}
$$


where $a$ averages the directivity gain which is a trivial work due to the fact that the directivity gain of each link is independent of other variables. Additionally, averaging over $r_{k}$ is also a simple work once we know the pdf of $r_{k}[10]$.

\section{B. Proof of Theorem 2}

The coverage probability can be expressed as

$$
\begin{aligned}
& \operatorname{Pr}\left(\bigcup_{k \in\{\mathrm{mu}, \mathrm{su}, \mathrm{sm}\}} \operatorname{SINR}_{k}\left(x_{k}\right)>\gamma_{k}\right) \\
& \quad=\sum_{k \in\{\mathrm{mu}, \mathrm{su}, \mathrm{sm}\}} \mathbb{E}_{x_{k}, \mathbf{G}}\left[\operatorname{Pr}\left(\operatorname{SINR}_{k}\left(x_{k}\right)>\gamma_{k}\right)\right]
\end{aligned}
$$

where $\operatorname{Pr}\left(\operatorname{SINR}_{k}\left(x_{k}\right)>\gamma_{k}\right)$ is given by

$$
\begin{aligned}
& \operatorname{Pr}\left(\operatorname{SINR}_{k}\left(x_{k}\right)>\gamma_{k}\right)=\operatorname{Pr}\left(\frac{P_{k} G_{k} h_{x_{k}} x_{k}^{-\alpha_{k}}}{\sum_{j \in\{\mathrm{mu}, \mathrm{su}\}} I_{j}+N_{0}}>\gamma_{k}\right) \\
& \approx\left\{\begin{array}{l}
\operatorname{Pr}\left(\frac{P_{k} G_{k} h_{x_{k}} x_{k}^{-\alpha_{k}}}{N_{0}}>\gamma_{k}\right) \quad k=\mathrm{sm} \\
\operatorname{Pr}\left(\frac{P_{k} G_{k} h_{x_{k}} x_{k}^{-\alpha_{k}}}{\sum_{j \in\{\mathrm{mu}, \mathrm{su}\}} I_{j}}>\gamma_{k}\right) k \in\{\mathrm{mu}, \mathrm{su}\}
\end{array}\right.
\end{aligned}
$$

where the interference can be ignored when UE is in the coverage of mmWave SBS, i.e., $k=s m$ and the interference cannot be neglected when UE is in the coverage of sub-6 GHz SBS or MBS, i.e., $k \in\{\mathrm{mu}, \mathrm{su}\}$. The following is the discussion about the associated distance-based conditional $k$-tier coverage probability,

(1) If $k=\mathrm{sm}$,

$$
\begin{aligned}
\operatorname{Pr}\left(\frac{P_{k} G_{k} h_{x_{k}} x_{k}^{-\alpha_{k}}}{N_{0}}>\gamma_{k}\right) & =\operatorname{Pr}\left(h_{x_{k}}>\frac{\gamma_{k} N_{0}}{P_{k} G_{k} x_{k}^{-\alpha_{k}}}\right) \\
& =\exp \left(-\frac{\gamma_{k} N_{0}}{P_{k} G_{k} x_{k}^{-\alpha_{k}}}\right)
\end{aligned}
$$

(2) If $k=\mathrm{mu}$,su,

$$
\begin{aligned}
& \operatorname{Pr}\left(\frac{P_{k} G_{k} h_{x_{k}}\left\|x_{k}\right\|^{-\alpha_{k}}}{\sum_{j \in\{\mathrm{mu}, \mathrm{su}\}} I_{j}}>\gamma_{k}\right)=\mathbb{E}_{x_{k}}\left[\exp \left(-\frac{\gamma_{k} \sum_{z \in \Phi_{j}} P_{j} h_{z} z^{-\alpha_{s}}}{P_{k} x_{k}^{-\alpha_{s}}}\right)\right] \\
& \mathbb{E}_{x_{k}}^{!}\left[\exp \left(-\frac{\gamma_{k} \sum_{z \in \Phi_{j}} h_{z} z^{-\alpha_{s}}}{x_{k}^{-\alpha_{s}}}\right)\right]
\end{aligned}
$$

where $\mathbb{E}_{x_{k}}\left[\exp \left(-\frac{\gamma_{k} \sum_{z \in \Phi_{j}} P_{j} h_{z} z^{-\alpha_{s}}}{P_{k} x_{k}^{-\alpha_{s}}}\right)\right]$ and $\mathbb{E}_{x_{k}}^{!}\left[\exp \left(-\frac{\gamma_{k} \sum_{z \in \Phi_{j}} h_{z} z^{-\alpha_{s}}}{x_{k}^{-\alpha_{S}}}\right)\right]$ of PPP and PCP have been derived in [19], respectively.
Thus, the coverage probability is given by

$$
\begin{aligned}
& \operatorname{Pr}\left.\bigcup_{k \in\{\mathrm{mu}, \mathrm{su}, \mathrm{sm}\}} \operatorname{SINR}_{k}\left(x_{k}\right)>\gamma_{k}\right) \\
&= \mathbb{E}_{x_{k}}\left[\frac{\omega}{\pi} \exp \left(-\frac{\gamma_{k} N_{0}}{P_{k} G_{\max } x_{k}^{-\alpha_{k}}}\right)\right. \\
&+\left.\frac{\pi-\omega}{\pi} \exp \left(-\frac{\gamma_{k} N_{0}}{P_{k} G_{\min } x_{k}^{-\alpha_{k}}}\right)\right] \\
&+ \sum_{k \in\{\mathrm{mu}, \mathrm{su}\}} \mathbb{E}_{x_{k}}\left[\exp \left(-\frac{\gamma_{k} \sum_{z \in \Phi_{j}} P_{j} h_{z} z^{-\alpha_{s}}}{P_{k} x_{k}^{-\alpha_{s}}}\right)\right] \\
& \mathbb{E}_{x_{k}}^{!}\left[\exp \left(-\frac{\gamma_{k} \sum_{z \in \Phi_{j}} h_{z} z^{-\alpha_{s}}}{x_{k}^{-\alpha_{s}}}\right)\right]
\end{aligned}
$$

where the first term can be calculated by averaging over $x_{k}$. The pdf of $x_{k}$ is given by Corollary 1 . $\mathbb{E}_{x_{k}}\left[\exp \left(-\frac{\gamma_{k} \sum_{z \in \Phi_{j}} P_{j} h_{z} z^{-\alpha_{S}}}{P_{k} x_{k}^{-\alpha_{S}}}\right)\right]$ and $\mathbb{E}_{x_{k}}^{!}\left[\exp \left(-\frac{\gamma_{k} \sum_{z \in \Phi_{j}} h_{z} z^{-\alpha_{S}}}{x_{k}^{-\alpha_{S}}}\right)\right]$ have been derived in [19].

\section{Abbreviations}

5G: the fifth generation mobile communication network; BP: Binary power; CSI: Channel state information; GP: Geometric programming; HetNets: Heterogeneous networks; LOS: Line-of-sight; MBS: Macro base station; MIMO: Multiple-input multiple-output; mmWave: Millimeter wave; NLOS: Non-line-of-sight; PCP: Poisson cluster point; PGF: Probability generating function; PPP: Poisson point process; QoS: Quality of service; SBS: Small-cell base station; SINR: Signal to interference plus noise ratio; UE: User

\section{Funding}

This work is supported by the National Natural Science Foundation of China (No. 61701037 and 61601503), Beijing Natural Science Foundation (No. L172033) and the 111 Project of China (B16006).

\section{Availability of data and materials}

The simulation data supporting this paper can be found and part source files can be shared.

\section{Authors' contributions}

The idea of this work was proposed by XZ and YL. YW performed the simulation. XZ wrote the manuscript. JB has given critical revision and helped revise the manuscript. All authors read and approved the final manuscript.

\section{Competing interests}

The authors declare that they have no competing interests.

\section{Publisher's Note}

Springer Nature remains neutral with regard to jurisdictional claims in published maps and institutional affiliations.

\section{Author details}

${ }^{1}$ National Engineering Laboratory for Mobile Network Technologies, Beijing University of Posts and Telecommunications, 100876 Beijing, China. ${ }^{2}$ Air Force Engineering University, 710051 Xi'an, China.

Received: 31 December 2016 Accepted: 7 February 2019

Published online: 26 February 2019 


\section{References}

1. J.G. Andrews, et al., What will 5 G be? IEEE J. Sel. Areas Commun. 32(6), 1065-1082 (2014)

2. F. Boccardi, et al., Five disruptive technology directions for 5G. IEEE Commun. Mag. 52(2), 74-80 (2014)

3. M. Di Renzo, Stochastic geometry modeling and analysis of multi-tier millimeter wave cellular networks. IEEE Trans. Wirel. Commun. 14(9), 5038-5057 (2015)

4. J.G. Andrews, et al., Modeling and analyzing millimeter wave cellular systems. IEEE Trans. Commun. 65(1), 403-430 (2017)

5. L. Wei, et al., Key elements to enable millimeter wave communications for 5G wireless systems. IEEE Wirel. Commun. 21(6), 136-143 (2014)

6. T. Bai, et al., Coverage and rate analysis for millimeter-wave cellular networks. IEEE Trans. Wirel. Commun. 14(2), 1100-1114 (2015)

7. S. Singh, et al., Tractable model for rate in self-backhauled millimeter wave cellular networks. IEEE J. Sel. Areas Commun. 33(10), 2196-2211 (2015)

8. A. Ghosh, et al., Millimeter-wave enhanced local area systems: a high-data-rate approach for future wireless networks. IEEE J. Sel. Area Commun. 32(6), 1152-1163 (2014)

9. H. Elshaer, et al., Downlink and uplink cell association with traditional macrocells and millimeter wave small cells. IEEE Trans. Wirel. Commun. 15(9), 6244-6258 (2016)

10. M. Di Renzo, in IEEE International Conference on Communications (ICC). Stochastic geometry modeling and performance evaluation of mmWave cellular communications, (London, 2015), pp. 5992-5997

11. M. N. Kulkarni, A. Ghosh, J. G. Andrews, A comparison of MIMO techniques in downlink millimeter wave cellular networks with hybrid beamforming. IEEE Trans. Commun. 64(5), 1952-1967 (2016)

12. F. W. Vook, et al., in IEEE 48th Asilomar Conference on Signals, Systems and Computers. Massive MIMO for mmWave systems, (Pacific Grove, 2014), pp. $820-824$

13. A. Adhikary, et al., Joint spatial division and multiplexing for mm-wave channels. IEEE J. Sel. Areas Commu. 32(6), 1239-1255 (2014)

14. M. Haenggi, et al., Stochastic geometry and random graphs for the analysis and design of wireless networks. IEEE J. Sel. Areas Commu. 27(7), 1029-1046 (2009)

15. R. W. Heath, M. Kountouris, T. Bai, Modeling heterogeneous network interference using Poisson point processes. IEEE Trans. Signal Pro. 61(16), 4114-4126 (2013)

16. H. S. Dhillon, et al., Modeling and analysis of K-tier downlink heterogeneous cellular networks. IEEE J. Sel. Area Commu. 30(3), 550-560 (2012)

17. S. Mukherjee, Distribution of downlink SINR in heterogeneous cellular networks. IEEE J. Sel. Area Commu. 30(3), 575-585 (2012)

18. N. Deng, W. Zhou, M. Haenggi, Heterogeneous cellular network models with dependence. IEEE J. Sel. Area Commu. 33(10), 2167-2181 (2015)

19. Y. J. Chun, M. O. Hasna, A. Ghrayeb, Modeling heterogeneous cellular networks interference using poisson cluster processes. IEEE J. Sel. Area Commu. 33(10), 2182-2195 (2015)

20. T. Bai, et al., in Global Conference on Signal and Information Processing (GlobalSIP). Coverage analysis for millimeter wave cellular networks with blockage effects, (Austin, 2013), pp. 727-730

21. R. K. Ganti, M. Haenggi, Interference and outage in clustered wireless ad hoc networks. IEEE Trans. Inform. Theory. 55(9), 4067-4086 (2009)

22. G. J. Foschini, Z. Miljanic, A simple distributed autonomous power control algorithm and its convergence. IEEE Trans. Vel. Technol. 42(4), 641-646 (1993)

23. N. Lee, et al., Power control for D2D underlaid cellular networks: modeling, algorithms, and analysis. IEEE J. Sel. Area Commun. 33(1), 1-13 (2015)

24. C. Guo, et al., -Fair power allocation in spectrum-sharing networks. IEEE Trans. Veh. Technol. 65(5), 3771-3777 (2016)

25. A. Gjendemsj, et al., Binary power control for sum rate maximization over multiple interfering links. IEEE Trans. Wirel. Commun. 7(8), 3164-3173 (2008)

26. $\mathrm{H}$. Alzer, On some inequalities for the incomplete gamma function. Math Comput. Am. Math. Soc. 66(218), 771-778 (1997)

27. A. Thornburg, T. Bai, R. Heath, Performance analysis of mmWave ad hoc networks. IEEE Trans. Signal. Process. 64(15), 4065-4079 (2016)

\section{Submit your manuscript to a SpringerOpen ${ }^{\circ}$ journal and benefit from:}

- Convenient online submission

- Rigorous peer review

- Open access: articles freely available online

- High visibility within the field

- Retaining the copyright to your article

Submit your next manuscript at $\$$ springeropen.com 